\title{
A Rare Case of Congenital Orbital Cystic Neuroblastoma in an Infant
}

\author{
Abolfazl Kasaee Seyed Mohsen Rafizadeh Hadi Ghadimi Kianoosh Shahraki \\ Fahimeh Asadi Amoli Mansooreh Jamshidian-Tehrani \\ Eye Research Center, Farabi Eye Hospital, Tehran University of Medical Sciences, Tehran, Iran
}

\section{Established Facts}

- Congenital cystic neuroblastoma is extremely rare, with only 3 cases of extra-adrenal congenital cystic neuroblastoma previously reported (2 in the thorax and 1 in the presacral region).

- No case of orbital involvement in congenital cystic neuroblastoma has been reported to date.

\section{Novel Insights}

- This is the first report of congenital cystic neuroblastoma involving the orbit.

- Successful resection of this large mass is discussed as a challenging surgery.

\section{Keywords}

Congenital neuroblastoma - Cystic neuroblastoma .

Neuroblastoma. Orbital neoplasm

\begin{abstract}
Neuroblastoma, a relatively common tumor in infants, is rarely present at birth. A solid mass is the typical appearance of this neoplasm, while cystic formation is uncommon. Congenital cystic neuroblastoma is extremely rare and no previous report exists of its occurrence in the orbit. A newborn girl was referred with an extremely large mass on the right side of her face on the third day of her life. Prenatal ultrasonography had revealed a large mass $(80 \times 80 \mathrm{~mm})$ in the frontal area. MRI revealed a heterogeneous mass composed of cystic and solid, contrast-enhancing components. Excisional biopsy was successfully performed and histopathologic diagnosis of neuroblastoma was confirmed. Systemic workup was negative for involvement of other organs and levels of
\end{abstract}

(c) 2018 S. Karger AG, Basel catecholamine metabolites of serum and urine (homovanillic acid and vanillylmandelic acid) were normal. Postoperative course was uneventful with complete healing of the anophthalmic socket and normal physical and mental growth and development of the infant. To our knowledge, this is the first report of congenital cystic neuroblastoma primarily arising in the orbit.

(c) 2018 S. Karger AG, Basel

\section{Introduction}

Neuroblastoma is the most common solid tumor in infancy and comprises about $20 \%$ of all tumors in this age group. Congenital neuroblastoma is uncommon, and fewer than 100 cases have been reported to date $[1,2]$.

Neuroblastoma usually presents with a solid mass. Cystic formation of neuroblastoma is rarely described. The majority of cases of cystic neuroblastoma are located in the adrenal glands and are rarely seen in other organs

\section{KARGER}

E-Mail karger@karger.com

www.karger.com/oop
Mansooreh Jamshidian-Tehrani, MD

Eye Research Center, Farabi Eye Hospital

Qazvin Square

Tehran 1336616351 (Iran)

E-Mail mansooreh_2468@yahoo.com 

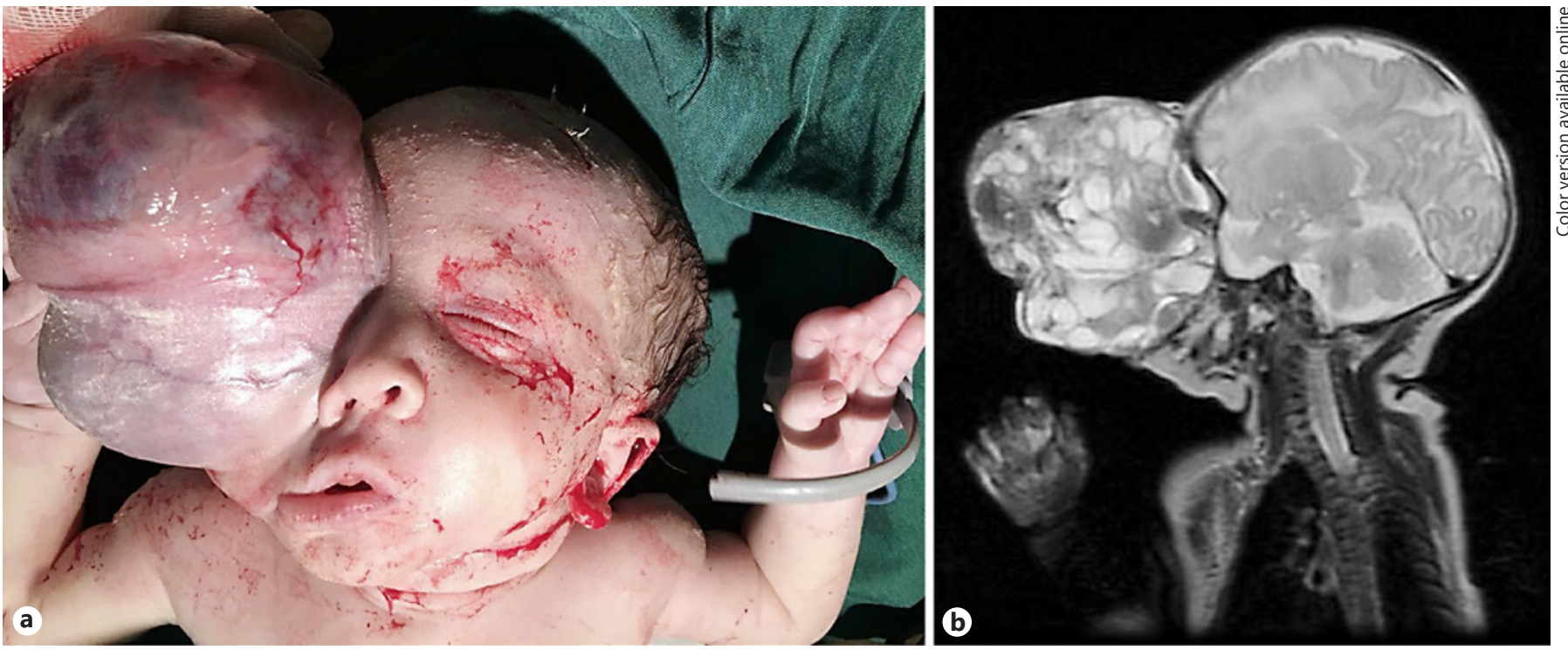

Fig. 1. a Clinical photograph of the patient showing huge right orbital mass with cystic and hemorrhagic appearance. $\mathbf{b}$ MRI showing gadolinium enhancement by the solid component of the lesion in sagittal section.

$[3,4]$. We report an extremely unusual case of congenital solitary orbital cystic neuroblastoma confirmed by immunohistochemical (IHC) studies in a newborn girl.

\section{Case Report}

A 3-day-old girl was referred to our hospital with a large orbital mass on the right side. The right globe and ocular adnexa were not seen (Fig. 1a). Examination of the left eye was normal. No other abnormality was reported by her pediatrician in routine physical examination on the day of birth.

Maternal and familial history were unremarkable. She was the first child of a healthy 28 -year-old mother, delivered by cesarean section at 39 weeks of gestational age. In prenatal ultrasonography performed on the $37 \mathrm{th}$ week of gestational age, the radiologist reported a large $(80 \times 80 \mathrm{~mm})$ mass in the forehead of the fetus that lacked active vascularity and originated from the orbit or skull. Earlier prenatal sonographic reports were not available.

MRI showed a large $(82 \times 66 \times 86 \mathrm{~mm})$ right orbital mass with solid and cystic components associated with expansion of bony orbital fossa and severe nasal deviation. After contrast injection, there was marked enhancement of the solid component of the lesion. Hemorrhagic component was detected within the lesion. The right globe could not be seen separately and there was no obvious intracranial extension of the mass (Fig. 1b). Routine laboratory tests including complete blood cell count and serum creatinine were within the normal range.

The study protocol was approved by the ethics committee of our institute and adhered to the tenets of the Declaration of Helsinki. After the patient's parents were informed about the thera- peutic options and informed consent for the procedure and for possible publication of the patient's photographs, imaging, and pathologic findings was obtained, an excisional biopsy was performed. The tumor, which was removed almost completely, was a circumscribed lobulated mass, covered by skin on the surface, with dimensions of $75 \times 60 \times 55 \mathrm{~mm}$. Cut sections showed solid components and cystic spaces filled with blood clot. Histopathologic study revealed a cystic mass composed of islands of small, round and oval tumoral cells with foci of rosette formation in a fibrillary stroma. Inconspicuous nucleoli and scant cytoplasm arranged in diffuse solid patterns were seen, accompanied by some vascular channels. Mitosis was rare and necrosis was not evident. Cystic spaces and marked hemorrhagic areas were visible (Fig. 2a, b). The tumor cells were diffusely immunoreactive for neuronspecific enolase (NSE), vimentin, GFAP, and S100 (positive reaction in stroma and some scattered tumoral cells) (Fig. 2c-e). However, immunostaining for actin, SMA, myogenin, p63 desmin, CD34, chromogranin, synaptophysin, and CD99 yielded negative results. Based on the morphologic features, IHC findings, and macroscopic cystic pattern, the diagnosis of cystic neuroblastoma was made.

After confirmation of the diagnosis by a second pathologist, systemic workup was completed. MRI of the brain, chest, abdomen, and pelvis and whole body MIBG scan showed no evidence of mass or malignancy in the adrenal glands and other body organs. Bone marrow aspiration and bone marrow biopsy were negative for tumoral cells. Levels of serum homovanillic acid and urine vanillylmandelic acid were normal. At the follow-up visit 6 months after surgery, complete healing of the anophthalmic socket had occurred and the patient's growth and development were normal (Fig. 3). 


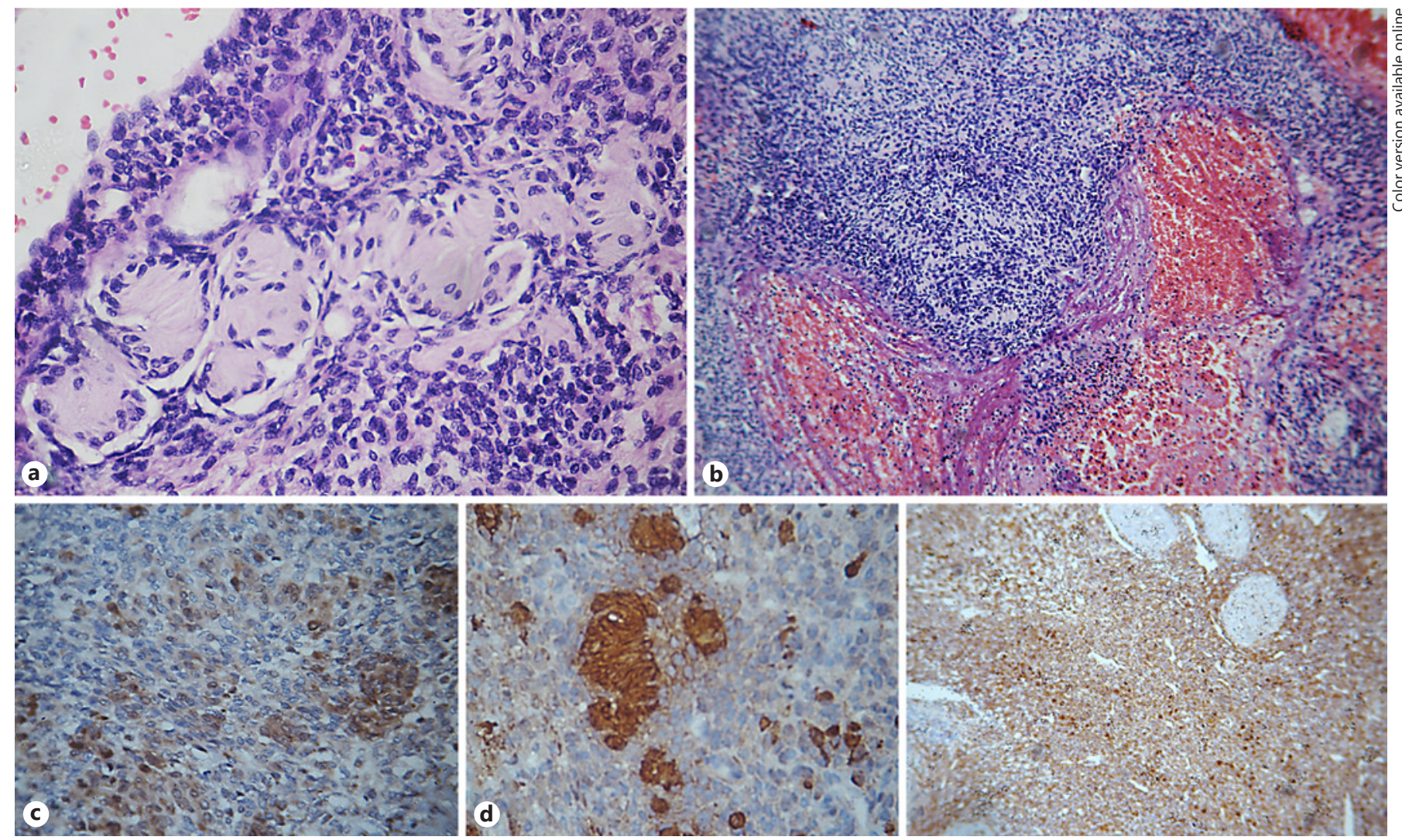

Fig. 2. a Microscopic study of the tumor, revealing small round cells with foci of rosette formation in a fibrillary stroma. H\&E stain. Magnification, $\times 100$. b Hemorrhagic areas and cystic spaces are seen within the mass. Immunohistochemical staining revealing diffuse positivity for NSE (c), focal positivity for S100 (d), and diffuse positivity for vimentin (e).

\section{Discussion}

Neonatal tumors are uncommon and comprise only $2 \%$ of pediatric malignant tumors. The most common neonatal tumor is neuroblastoma, accounting for approximately $20 \%$ of all tumors in this age group [1]. Neuroblastoma often arises in the adrenal glands or sympathetic ganglia and common locations for metastases are the liver and retroperitoneal lymph nodes. Orbital metastasis has been reported in $10-20 \%$ of childhood neuroblastomas and often presents with proptosis and periorbital ecchymosis. The age at presentation is usually 15 months or later. Congenital neuroblastoma is rare, and fewer than 100 cases have been reported in the literature $[2,5,6]$.

Neuroblastomas often contain solid components but cystic formation is rarely encountered. Cystic neuroblasto$\mathrm{ma}$, a rare variant, often presents at a lower age and is associated with lower rates of metastasis. Thus, its long-term prognosis is more favorable [3]. In our patient, in spite of the

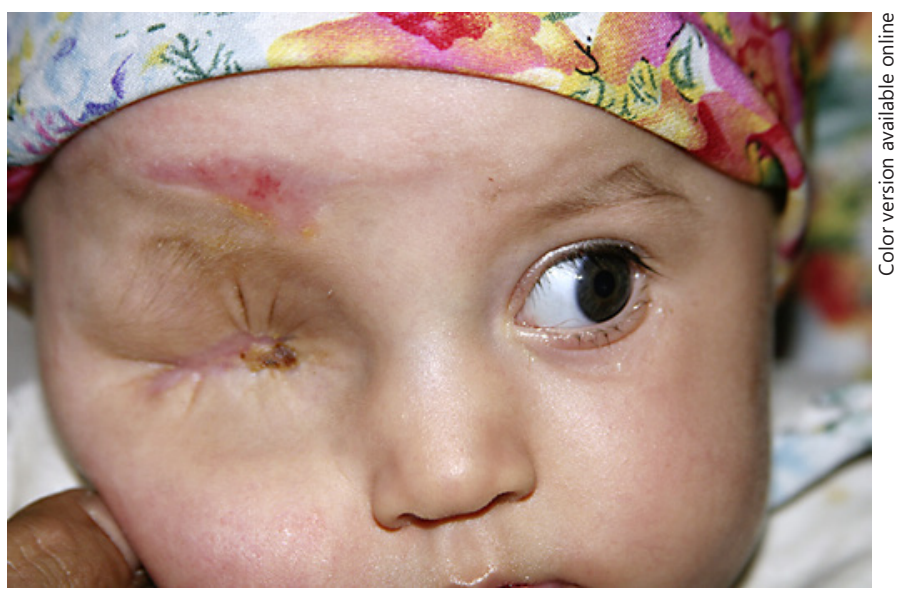

Fig. 3. Healing of the anophthalmic socket 6 months after surgery. 
dramatic presentation of the large tumor, workup for remote metastasis was negative and there was no recurrence.

The most common site for cystic neuroblastoma is the adrenal glands, and extra-adrenal occurrence is extremely unusual, with only 3 cases being previously reported in the English literature, including 2 in the thorax and 1 in the presacral region $[3,4]$. Additionally, 2 cases of congenital orbital teratoma with neuroblastic features have been reported so far. An orbital teratoma with neuroblastic and rhabdomyoblastic elements has been reported by Soares et al. [7]. The second case, reported by Wilson et al. [8], was an orbital teratoma with malignant neuroblastic cells. However, to our knowledge, there is no previous report of congenital cystic neuroblastoma within the orbit without any systemic involvement.

Cystic neuroblastomas often contain solid and cystic components. Classic pathologic findings include diffuse cystic formation lined by thick fibrous walls with scattered clusters of neuroblastic cells adjacent to areas of hemorrhage and necrosis, but calcification is rare [6].

In fact, necrosis and hemorrhage are often reported in both solid and cystic neuroblastoma. However, calcification, which is seen in most cases of solid neuroblastoma, is rarely present in cystic neuroblastoma $[3,4]$.

In our case the preoperative differential diagnosis was wide, including rhabdomyosarcoma, Ewing sarcoma/ primitive neuroectodermal tumors, medulloblastoma, small cell osteosarcoma, lymphoblastic lymphoma, blastomatous Wilm's tumor, and small cell desmoblastic tumor [9]. Histopathologic examination and IHC study limited our final differential diagnosis.

The tumor cells in neuroblastoma are immunohistochemically positive for neuronal markers such vimentin and NSE, but negative for CD45 and CD99/MIC2, both of which are characteristic of primitive neuroectodermal tumors.
A variety of markers can be studied in neuroblastoma, many of which lack sensitivity, specificity, or both as individual reagents and must be used as panels. NSE is present in $85-100 \%$ of cases of neuroblastoma. Chromogranin is a more specific marker, but is less sensitive (present in 52\% of cases). In our case NSE and chromogranin showed a positive and negative reaction, respectively. Markers that are nonreactive in neuroblastoma are thus useful in the recognition of other round cell malignant neoplasms [9].

In our case the postoperative serum and urine levels of catecholamines were normal. Measurement of catecholamine levels in the follow-up of cystic neuroblastoma patients is not necessary because elevation of serum and urine catecholamine is rarely seen due to the paucity of solid components in this type of tumor. Solid infantile neuroblastomas, similarly, are uncommonly associated with a rise of catecholamine levels $[3,10]$. The reason is the small volume of tumor, with incompletely differentiated cells in infantile neuroblastoma.

In conclusion, this patient, who is the first case of congenital cystic neuroblastoma diagnosed in the orbit, underwent successful surgical management and had an uneventful postoperative course.

\section{Statement of Ethics}

The study protocol was approved by the ethics committee of our institute and adhered to the tenets of the Declaration of Helsinki. The patient's parents gave us permission to use any of her photographs and a copy of the consent is on file.

\section{Disclosure Statement}

The authors declare no conflicts of interest. No grants or funding were received for this study.

\section{References}

1 Bader JL, Miller RW. US cancer incidence and mortality in the first year of life. Am J Dis Child. 1979 Feb;133(2):157-9.

2 Sul HJ, Kang D. Congenital neuroblastoma with multiple metastases: a case report. J Korean Med Sci. 2003 Aug;18(4):618-20.

3 D'Alessio A, Piro E, Beretta F, Abati L, Brugnoni M, Assi A et al. Presacral cystic neuroblastoma: case report and review of the literature. J Pediatr Hematol Oncol. 2006 Aug; 28(8):534-6.

4 Richards ML, Gundersen AE, Williams MS. Cystic neuroblastoma of infancy. J Pediatr Surg. 1995 Sep;30(9):1354-7.
5 Ahmed S, Goel S, Khandwala M, Agrawal A, Chang B, Simmons IG. Neuroblastoma with orbital metastasis: ophthalmic presentation and role of ophthalmologists. Eye (Lond). 2006 Apr;20(4):466-70.

6 Atkinson GO Jr, Zaatari GS, Lorenzo RL, Gay BB Jr, Garvin AJ. Cystic neuroblastoma in infants: radiographic and pathologic features. AJR Am J Roentgenol. 1986 Jan;146(1):1137.

7 Soares EJ, da Silva Lopes K, De Souza Andrade J, Faleiro LC, Alves JC. Orbital malignant teratoma: a case report. Orbit. 1983;2(4): $235-42$.
8 Wilson DJ, Dailey RA, Wobig JL, Dimmig JW. Neuroblastoma within a congenital orbital teratoma. Arch Ophthalmol. 2002 Feb; 120(2):213-5.

9 Dabbs D. Diagnostic immunohistochemistry: Theranostic and genomic applications. 4th ed. Philadelphia: Saunders; 2013.

10 Cassady C, Winters WD. Bilateral cystic neuroblastoma: imaging features and differential diagnoses. Pediatr Radiol. 1997 Sep;27(9): 758-9. 